\title{
ROM: READ ONLY MEMORY
}

\section{Macarena Bernal}

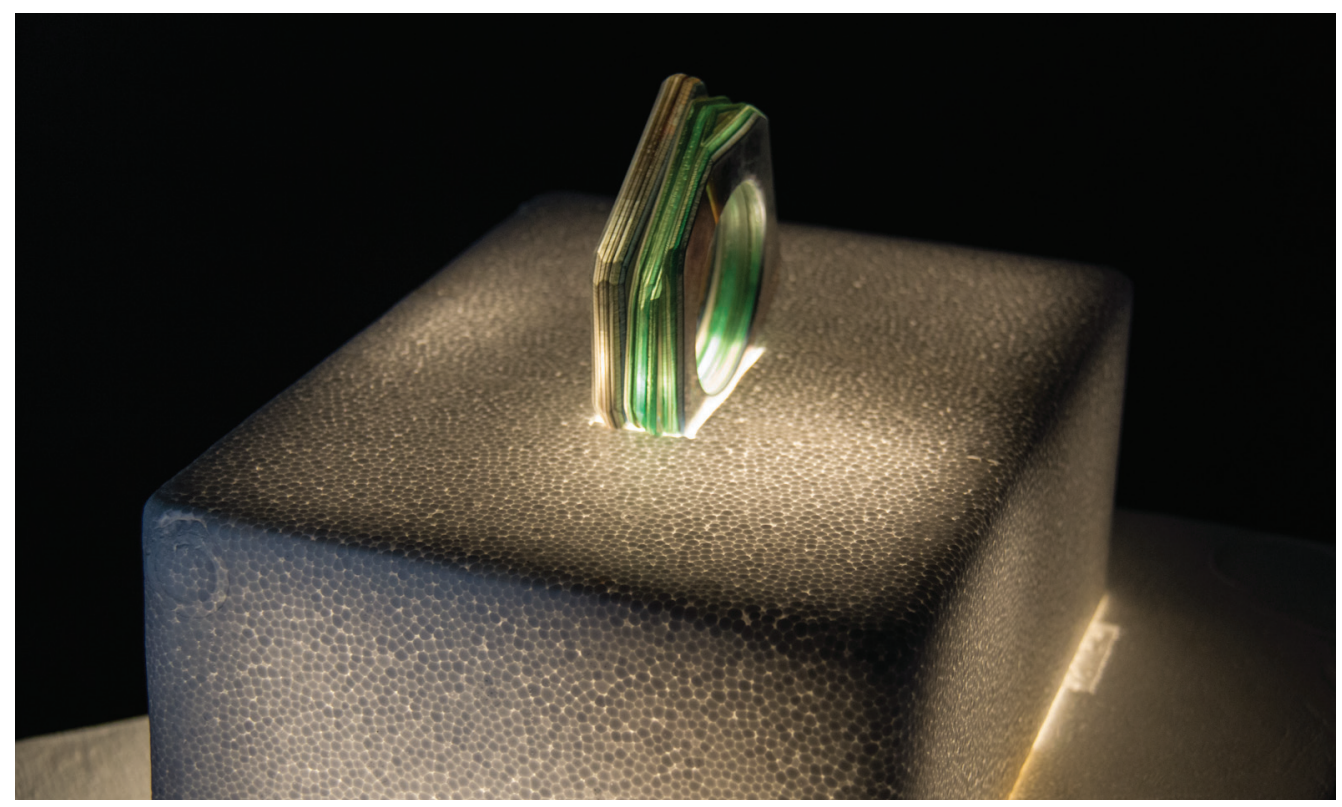

Figure I. Macarena Bernal, Rom: Read Only Memory, 20 I9, installation view.

Contemporary jewellery, being a young branch of the applied arts, has the difficult task of attracting an audience. Bearing the weight of history on its shoulders, this young artistic movement capitalises on its seductive nature $-\mathrm{a}$ distinctive conceptual tendency in which materials and skills are placed at the service of ideas.

Possibly the reason why I find it so attractive is that it offers me, an established jeweller, the chance to rebel. Owning this, I can demonstrate that I do not conform to the indoctrinating norms that a large portion of society ascribes to, and that I'm willing to see beyond the pre-existing hierarchy of materials and their affixed economic value.

Studying the physical characteristics that determine value in a material became a focal point for the research behind Rom: read only memory, my Master of Visual Arts project.

Rom is the name I gave a material I invented to capture and express my ideas in the form of contemporary jewellery and artifacts. It sits at the core of a speculative narrative I have generated about the preservation of information in the form of wearables and, most importantly, it purports to signify a new form of value. Not necessarily economic value, but more the divine kind because of its capacity to reflect and contain splendour, satisfying my personal urge to hold things that shine - which can be viewed a consequence of my cultural formation as a Latin American woman brought up in the 1980s and '90s in Chile. 


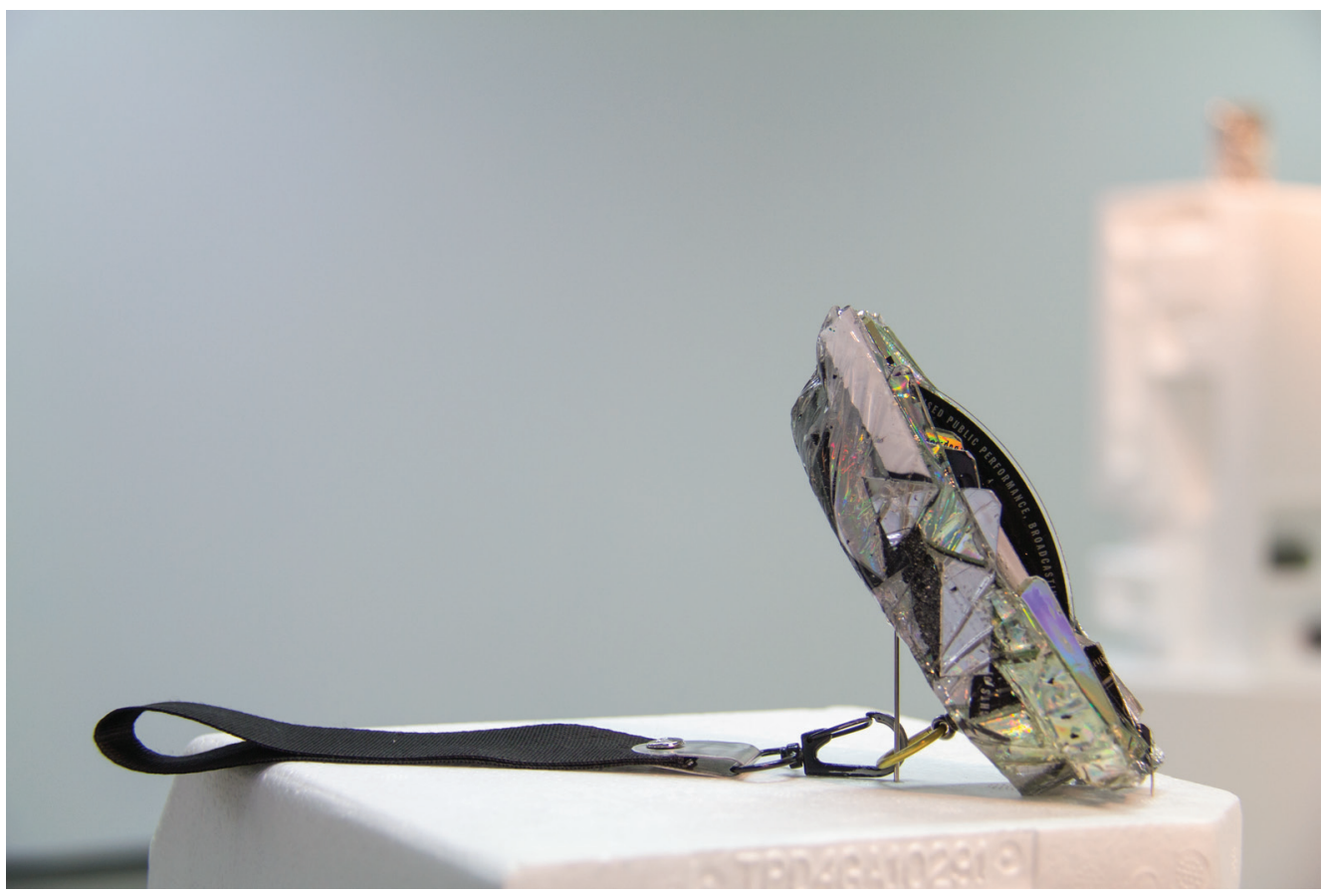

Figure 2. Macarena Bernal, Rom: Read Only Memory, 2019, installation view.

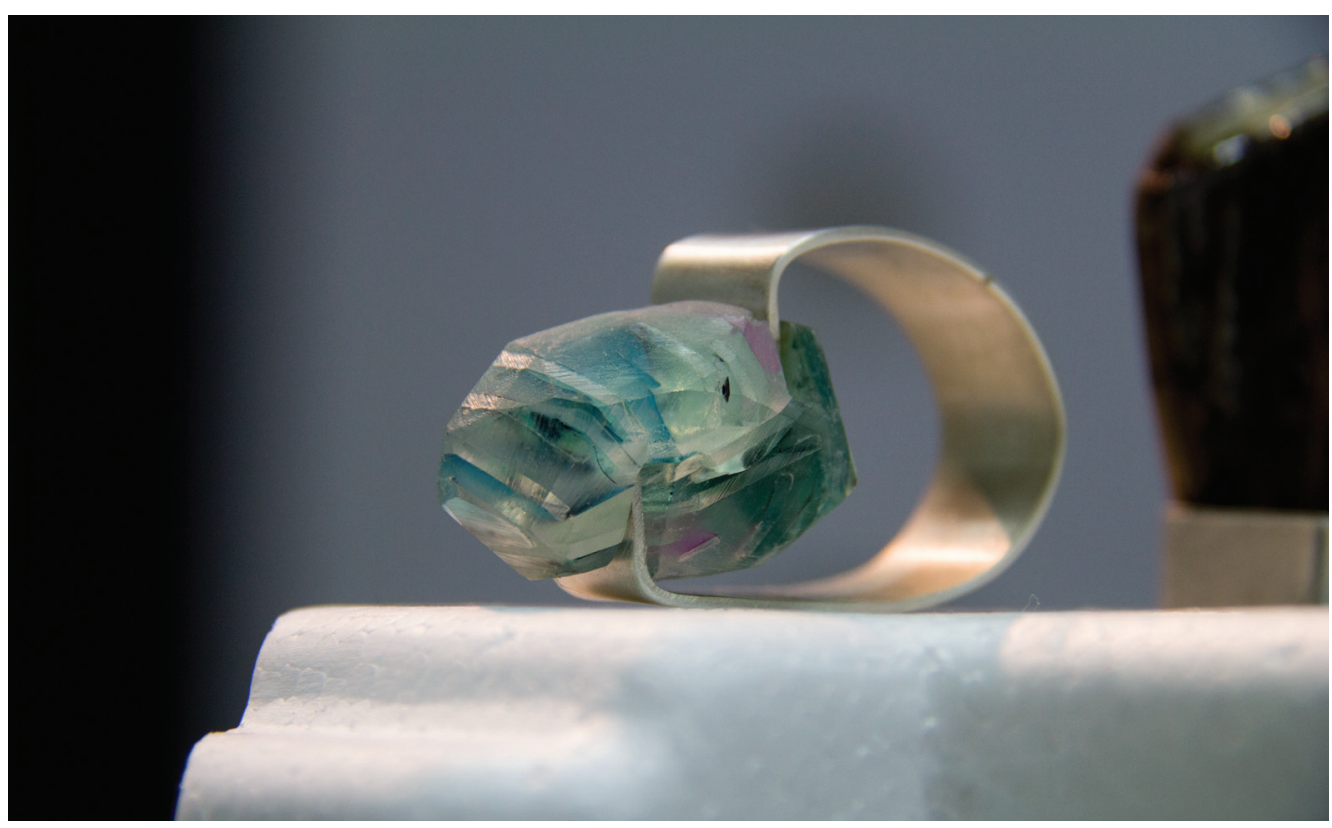

Figure 3. Macarena Bernal, Rom: Read Only Memory, 2019, installation view. 
Rom as a material originates from the amalgamation of discarded CD-ROMs, various other obsolete technologies, epoxy resin, and precious and semiprecious metals. Purportedly because of the high-value information contained within Rom, jewellery made from it could be considered wearable archives. Thus, the possibility of such information being recovered by researchers in a distant future, if the right technology was made available, could theoretically be plausible.

Early on, I resorted to using discarded packaging as moulds for some of the bracelets and earrings in the project, allowing me to imprint them with the appearance of techy artifacts. This led me to the idea of capitalising on the close relationship between jewellery and techy artifacts for the exhibition, devising a display system made from polystyrene packaging retrieved from electronics stores' waste bins. For me it was another example of imprinted memory. The negative spaces pre-formed on the polystyrene read as clues to an artefact's prior life, complementing the narrative for a series of contemporary jewellery pieces and artifacts that turned out to be something of a personal archive of memories, thus emphasising the title of the exhibition, "Rom:

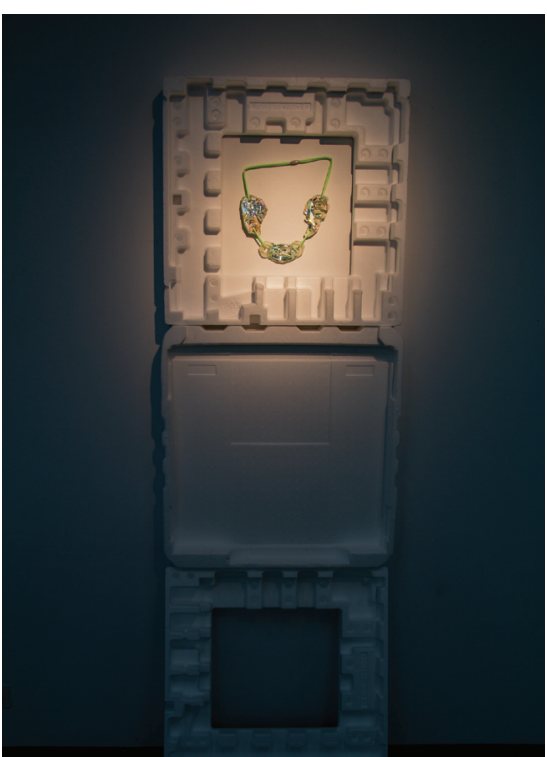

Figure 4. Macarena Bernal, Rom: Read Only Memory, 2019 , installation view. read only memory."

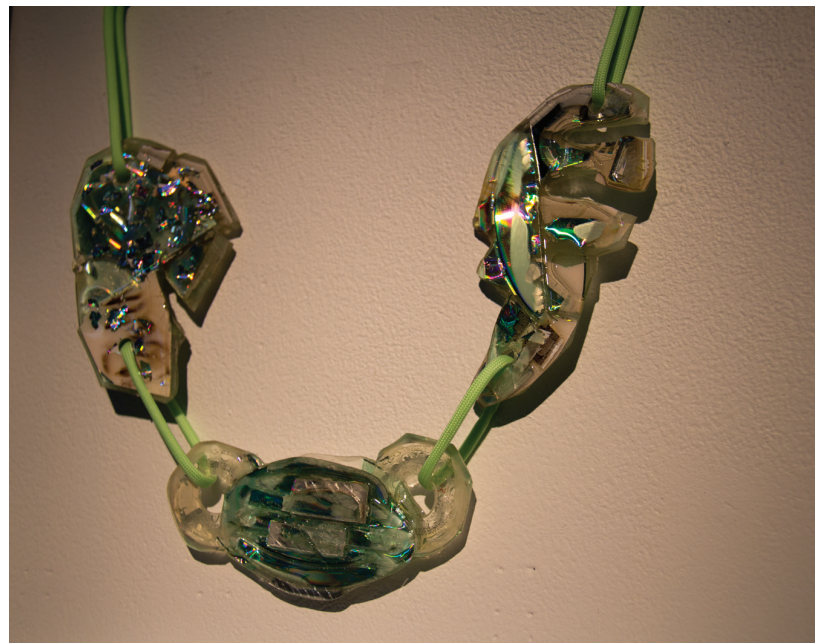

Figure 5. Macarena Bernal, Rom: Read Only Memory, 2019, installation view.

A a Chilean national, Macarena Bernal has been a New Zealand citizen since 2018. She has a bachelor's degree in visual arts from Finis Terrae University in Santiago, Chile, an advanced diploma in jewellery practice from MIT, and now an Master of Visual Arts from the Dunedin School of Arts in New Zealand. Her research focuses on preservation and responsibility, passing on valuable information to future generations, and dealing with relevant issues like communication, obsolete technology and archiving necessary for the survival of cultures.

Image Credits: Photographs by Pam McKinlay 